\title{
LIGHT EMISSION BY SEMICONDUCTOR NANOSTRUCTURES IN DIELECTRIC MEDIUM OR CLOSE TO PLANE INTERFACE
}

\author{
P. LAVAllard \\ Groupe de Physique des Solides, CNRS URA 17, Universités Paris VI et VII \\ Tour 23, 2 place Jussieu, 75251 Paris Cedex 05, France
}

\begin{abstract}
We review several situations in which the emission rate of a nanostructure is modified by its environment. We first consider small objects embedded in media with different refractive indices. The local electromagnetic field and the rate of spontaneous emission of the nanostructure are enhanced or inhibited by the induced dipole charges on the interface. In quantum wells or nanocrystals embedded in a low dielectric constant medium, the binding energy of excitons is increased. In anisotropic microcrystals, the local field is anisotropic and emission of light is polarized. We consider especially the case of $p^{+}$-doped porous silicon in which nanocrystals are elongated. Another interesting situation occurs when a dipole is in the vicinity of a dielectric interface. The local field acting on the dipole results from the interferences of incident and reflected beams. Contributions from evanescent waves are important when the dipole is located in the medium of low refractive index, very close to the surface. The intensity of emission and its pattern are modified by the vicinity of the dielectric interface. Close to the interface semiconductor/air, the exciton binding energy is increased. In the vicinity of a metallic surface, a nonradiative transfer to the metal occurs for small distances of the nano-object or quantum well to the surface and the quantum efficiency varies with the distance to the metallic surface. The exciton binding energy of a quantum well is decreased close to a metallic surface.

PACS numbers: 78.20.Bh, 78.55.Cr, 78.55.Et
\end{abstract}

\section{Introduction}

The rate of spontaneous emission of a dipole is not an intrinsic property of the dipole but depends on its environment [1]: dielectric medium [2-5], dielectric interface [6-8], metallic interface $[9,10]$ or cavity [11]. The modification of the emission rate of a dipole has been studied theoretically for many years. Numerous experimental studies have been done either with fluorescent ions or dye molecules [12-17]. In semiconductor physics the effect of a cavity on the rate of spontaneous emission of a quantum well was recognized recently $[18,19]$, and during the last years, only a few studies have been done on the effect of a dielectric 
environment [4] or a close interface [20-24] on the optical properties of nanostructures. There is, to our knowledge, no comprehensive review of all the situations in which the spontaneous emission of a nanocrystal (NC) or a quantum well (QW) is modified by its environment. It is the purpose of this lecture to review these situations.

As the emission of a QW or a NC is well described as a dipole emission, most of the conclusions, which could be obtained from the previous studies done on ion or molecule emission, apply to semiconductor nanostructure emission. However, in these studies, the transition dipole was generally considered as a constant and only its coupling with light was expected to vary with the environment. This is justified since the length of the dipole in atoms or molecules, only a few angstroms, is much smaller than the distance of the dipole to any material body which has to be considered in an experiment. The situation is different for excitons in a semiconductor [25-29]. The length of the equivalent dipole is given by the size of the envelope function which is usually larger than the exciton Bohr radius. The envelope function size depends on the semiconductor and the size of the nanostructure. It is usually much larger (a few nanometers in III-V compounds) than the dipole length in atoms or molecules. With modern techniques one can currently grow QWs and barriers with an accuracy of one monolayer thickness. Situations in which the distance between QW or NC to the interface is comparable to the Bohr radius are easily obtained. The Coulomb interaction between electron and hole is then modified by the environment and the transition dipole is no more constant.

This paper is organized as follows. In part 2, Sec. 2.1, we review the coupling with light of a small object immersed in a bulk dielectric medium. Different objects are considered, spherical microcrystal, coated microcrystal, spheroid microcrystal and impurity in a spherical microcrystal. In Sec. 2.2, the modification of Coulomb interaction is evaluated for a QW or NC. In part 3, Sec. 3.1, we review the coupling with light of a small object close to a dielectric interface. The intensity and the direction of emission are considered. In Sec. 3.2 the modification of Coulomb interaction due to the presence of a dielectric interface is evaluated for a $\mathrm{QW}$. In part 4, Sec. 4.1, we review the coupling with light of a small object close to a mirror. Thick and thin mirrors are considered. In Sec. 4.2 the modification of Coulomb interaction due to the presence of a metallic interface is evaluated for a $\mathrm{QW}$. In conclusion we summarize the results obtained for a $\mathrm{QW}$ or $\mathrm{NC}$.

\section{A small object in a bulk dielectric medium*}

\subsection{Coupling with light}

\subsubsection{Spherical microcrystals}

Let us consider a luminescing object whose size is smaller than the wavelength of luminescence light. The object is embedded in a dielectric medium. We suppose that the transition is electric-dipole allowed and the dipole constant.

*The main results are summarized in Table $\mathrm{I}$. 
The modification of the dipole due to the dielectric medium will be considered in Sec. 2.2.

The rate of spontaneous emission is given by the Fermi "golden rule" [4]:

$$
\frac{1}{\tau_{\mathrm{R}}}=\frac{2 \pi}{\hbar}\left|\left\langle i\left|\mu E_{\text {int }}^{+}\right| f\right\rangle\right|^{2} \rho(\hbar \omega) \text {. }
$$

$\tau_{\mathrm{R}}$ is the radiative lifetime, $\mu$ is the material dipole operator, $E_{\text {int }}^{+}$is the electric field creation operator inside the luminescent material and $\rho(\hbar \omega)$ is the density of optical modes at the energy $\hbar \omega .|i\rangle$ and $|f\rangle$ are the initial and final states. Several of these quantities depend on the refractive indices of the small crystal $\left(n_{\text {int }}\right)$ and the dielectric medium $\left(n_{\text {ext }}\right)$.

1) Since the object is much smaller than the wavelength of light, the density of optical modes $\rho(\hbar \omega)$ is the density of free modes in the dielectric medium. It is proportional to $n_{\text {ext }}^{3}$.

2) The electric field creation operator in the surrounding medium is proportional to $n_{\text {ext }}^{-1}$ :

$$
E_{\text {ext }}^{+}=\frac{1}{n_{\text {ext }}}\left(\frac{2 \pi \hbar \omega}{V}\right)^{1 / 2} \mathrm{e}^{\mathrm{i} k \cdot r_{a^{+}}}
$$

where $a^{+}$is the photon creator operator, $k$ is the wave vector, $r-$ the position vector and $V$ - the normalization volume of the optical modes.

3) As the electromagnetic field is homogeneous inside the microcrystal the internal field can be calculated as in electrostatic theory by taking into account the depolarizing field due to the dipole charges on the interface

$$
E_{\text {int }}^{+}=\frac{3}{\left(n_{\text {int }}^{2} / n_{\text {ext }}^{2}+2\right)} E_{\text {ext }}^{+} \text {. }
$$

Substituting $E_{\text {int }}^{+}$and $\rho(\hbar \omega)$ in formula (2.1), one finds the rate of spontaneous emission of a small crystal

$$
\frac{1}{\tau_{\mathrm{R}}}=\left(\frac{3}{n_{\text {int }}^{2} / n_{\text {ext }}^{2}+2}\right)^{2} \frac{n_{\text {ext }}}{n_{\text {int }}} \frac{1}{\tau_{\mathrm{R}}^{0}},
$$

where $1 / \tau_{\mathrm{R}}^{0}$ is the rate of spontaneous emission for $n_{\mathrm{ext}} / n_{\mathrm{int}}=1$.

Let us consider a semiconductor NC with $n_{\text {int }}=3.5 \mathrm{immersed}$ in air. The spontaneous lifetime of an electron-hole pair is found to be increased by a factor 80 !

When the size of the sphere becomes comparable to the wavelength of light, the emission of light cannot be described as the emission in the free optical modes of the medium surrounding the sphere. On the contrary, emission occurs towards the modes localized in the sphere. Figure 1 shows the spontaneous emission rate of a $70 \AA$ quantum dot at the center of an $\mathrm{Al}_{0.4} \mathrm{Ga}_{0.6} \mathrm{Ag}$ dielectric sphere of radius $a$ [30]. In the limit $a \rightarrow 0$, the emission rate is found independent of $a$ and given by Eq. (2.1). When the size of the sphere is increased, emission is alternatively enhanced and inhibited. If the dipole is not in the center of the sphere, several sharp resonances appear. They are due to a coincidence between a dipole and an internal standing wave in the sphere.

It is worthwhile to note that, for a range of photon energy where the optical dielectric constant does not vary much, the curve, rate of emission versus 


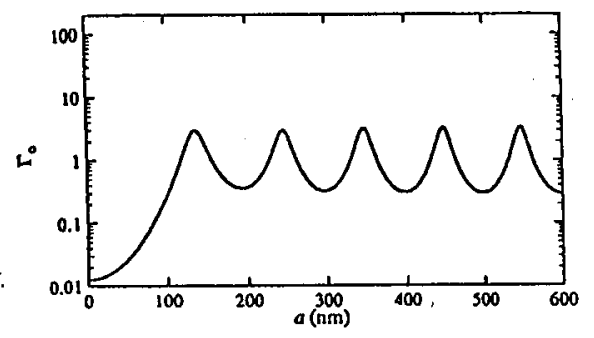

Fig. 1. Spontaneous emission rate of a $7 \mathrm{~nm}$ quantum well at the center of an $\mathrm{Al}_{0.4} \mathrm{Ga}_{0.6} \mathrm{As}$ dielectric sphere of radius $a$. The transition wavelength is $700 \mathrm{~nm}$. Reproduced with permission from Ref. [30]. Copyright by the American Institute of Physics.

radius, can be interpreted as giving the rate of emission versus $1 / \lambda$ for a given sphere size. Experiments have been done with GaAs microcrystals deposited on $\mathrm{Si}$ substrate and immersed in liquid nitrogen [31]. The size selected crystals exhibit modified photoluminescence spectra with blue shifts up to $10 \mathrm{meV}$. The size of the GaAs crystals excludes electronic quantum confinement effects. However, the crystal size is of the same range as the emission wavelength and the microcrystals form three-dimensional optical microcavities. A maximum of photonic density of states above the band-gap energy enhances the emission rate and then modifies the shape of the luminescence spectrum.

\subsubsection{Coated spherical microcrystals}

2.1.2.1. Dielectric coating. It has now become possible to grow composite $\mathrm{NCs}, \mathrm{CdS} / \mathrm{AgI}, \mathrm{CdS} / \mathrm{TiO}_{2}, \mathrm{ZnS} / \mathrm{CdSe}, \mathrm{ZnSe} / \mathrm{CdSe}, \mathrm{CdS} / \mathrm{PbS}$ [32]. In these materials not only the confinement energy of particles is modified but also the local field responsible for the emission of light. From electrostatic theory one easily finds the internal field as a function of the external field far from the coated particle

$$
E_{\text {int }}=\frac{9 \varepsilon \varepsilon_{2}}{\left(\varepsilon_{2}+2 \varepsilon\right)\left(\varepsilon_{1}+2 \varepsilon_{2}\right)+2 \Gamma\left(\varepsilon_{1}-\varepsilon_{2}\right)\left(\varepsilon_{2}-\varepsilon\right)} E_{\text {ext }} .
$$

$\varepsilon=n^{2}$ is the dielectric constant of the surrounding medium, $\varepsilon_{1}, \varepsilon_{2}$ are the dielectric constants of the nanoparticle and the coating medium respectively. $\Gamma$ is the volume ratio of the two concentric spheres. To calculate the emission rate of the coated microcrystal, the same analysis as for spherical microcrystals applies when replacing the internal field as given by formula (2.3) with the expression (2.5). A close system was studied recently [33]. It consists in water-in-oil micro-emulsions containing dye molecules. The micelles can be modeled as concentric dielectric spheres consisting of a water core and a surfactant layer with the dielectric constants $\varepsilon_{1}$ and $\varepsilon_{2}$. The photoluminescence lifetime of molecules is studied as a function of the optical dielectric constant of the solvent. Figure 2 shows a plot of the measured inverse lifetime as a function of the quantity $\left(E_{\text {int }} / E_{\text {ext }}\right)^{2} n$ which is the expected variation of the radiative lifetime. As the direct chemical environment of the molecule is not modified, one can assume that the nonradiative lifetime of the molecule is not affected by the solvent. The linear plot of Fig. 2 allows thus to determine both the nonradiative and the radiative lifetimes of the dye molecules. 


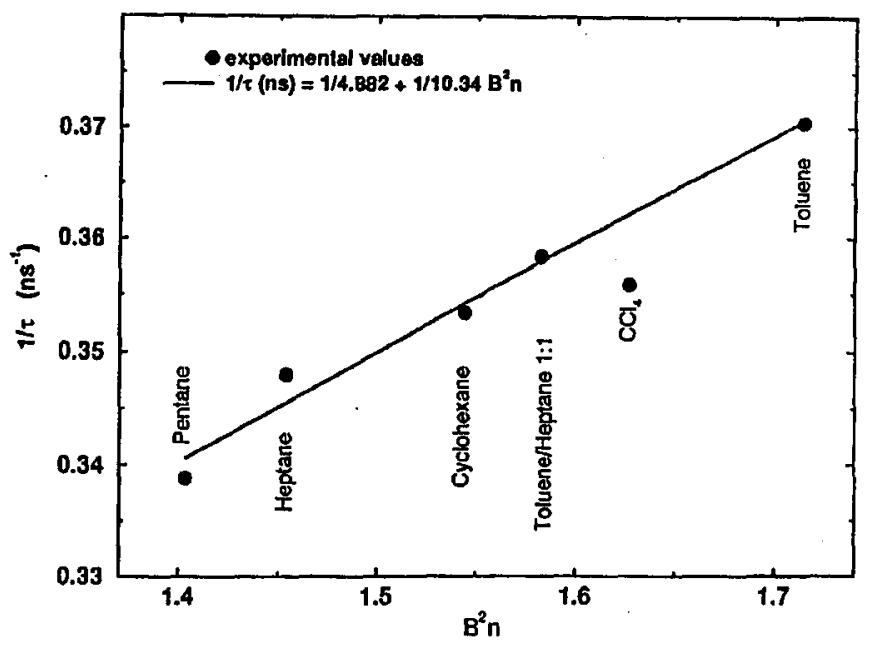

Fig. 2. Lifetime of sulforhodamine $B$ molecules in inverted micelles as a function of $B^{2} n . B$ is the ratio of the electromagnetic fields inside and outside the micelle (see Eq. (2.5)). $n$ is the refractive index of the solvent. The linear relation $1 / \tau$ (ns) $=1 / 4.882+B^{2} n / 10.34$ allows to determine the nonradiative lifetime $4.882 \mathrm{~ns}$ and the radiative lifetime $10.34 / B^{2} n$. From Ref. [33].

2.1.2.2. Metallic coating. The interest in metal-coated particles originates from the expectation of large linear and nonlinear optical responses due to the surface plasmon resonance. As seen in formula (2.3), an enhancement of the local field is indeed expected when $\varepsilon_{1}=-2 \varepsilon_{2}$. Figure 3 shows the field versus wa velength

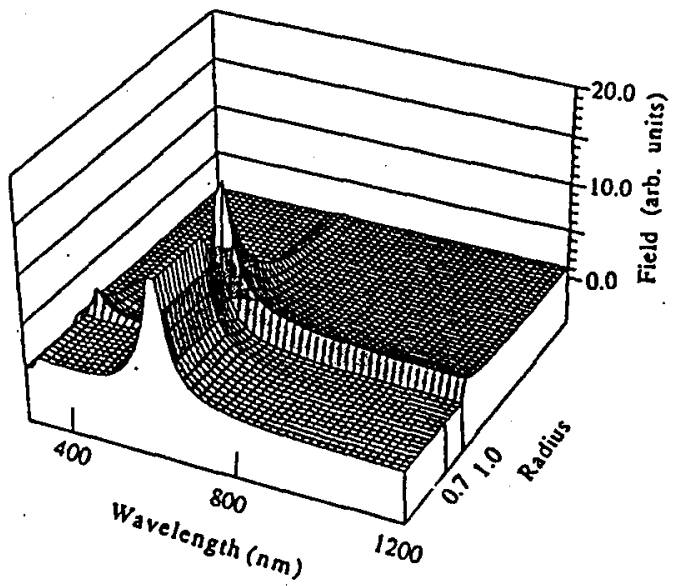

Fig. 3. Field versus wavelength and radius in Ag-coated CdS nanocrystal. The core-shell ratio is 0.7. Reproduced with permission from Ref. [34]. Copyright by the American Institute of Physics. 
and radius for CdS/Ag. An enhancement of the local field by a factor 10 is expected around $500 \mathrm{~nm}$, close to the surface plasmon resonance. Preliminary experiments on $\mathrm{Au}_{2} \mathrm{~S}$ particles coated with gold have been recently realized [35].

\subsubsection{Spheroidal microcrystals}

In non-spherical microcrystals, the local field is not parallel to the external electromagnetic field. For example, in an elliptical microcrystal, the ratio of the local and external fields in the $x$ direction is

$$
\left(\frac{E_{\text {loc }}}{E_{\text {exc }}}\right)_{x}=\frac{1}{1+\left(\frac{n_{\text {int }}^{2}}{n_{\text {ext }}^{2}}-1\right) A_{x}},
$$

where $A_{x}$ is the depolarizing factor for the $x$ direction. The depolarizing factors depend on the ellipticity of the microcrystal. They can be calculated from electrostatic theory [36].

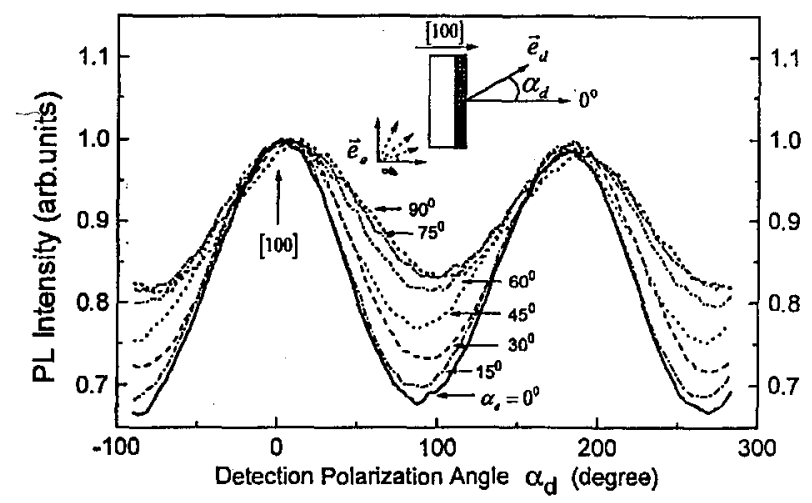

Fig. 4. Variation of the photoluminescence intensity with the angle of the detection polarizer with respect to the $[100]$ growth direction for different exciting light polarizations. Reproduced with permission from Ref. [38]. Copyright by the American Institute of Physics.

Let us consider porous silicon as an example $[37,38] . p$-type porous silicon is composed of NCs elongated mainly along the [100] direction. Figure 4 shows the intensity of photoluminescence as a function of the detection polarization angle when light propagates in the plane normal to the [100] direction. Whatever the excitation polarization is, the photoluminescence intensity is the largest when the direction of the detection polarization is parallel to the major axis of the NCs. This is expected since the depolarization factor is smaller for this direction. Figure 5 shows the degree of polarization of luminescence as a function of energy for light propagating along the [100] direction. For this direction, the silicon NCs appear randomly oriented and luminescence light is polarized as the excitation light. As the transverse component of the field is more attenuated than the longitudinal one, polarized light excites more the $\mathrm{NCs}$ which have their axes along or close to the direction of polarization. These NCs also emit light with preferential polarization along their axes and, as a consequence, the luminescence is partially polarized as 

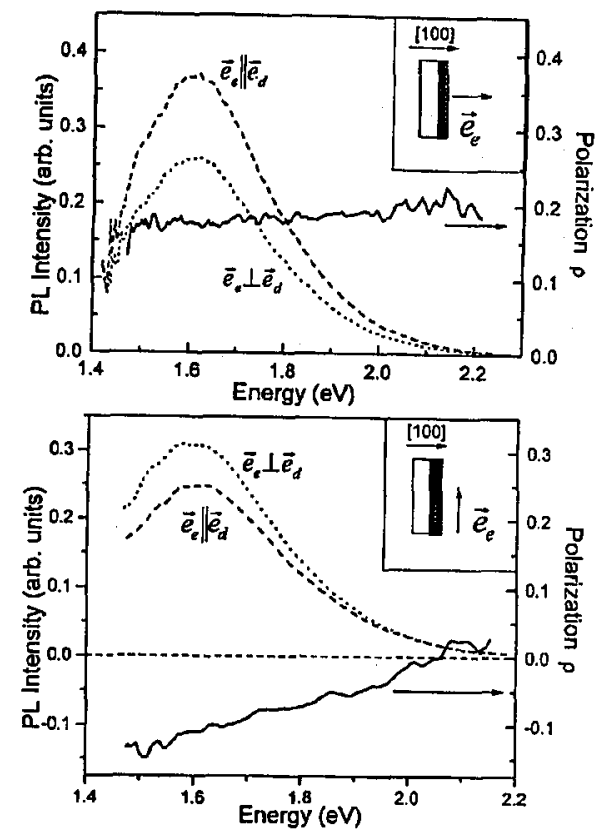

Fig. 5. Photoluminescence intensity detected with polarization parallel and perpendicular to that of the exciting light (dashed and dotted line, respectively) and degree of polarization $\rho$ as a function of detection energy: (a) excitation polarization parallel to the [100] growth direction, (b) excitation polarization perpendicular to [100]. Reproduced with permission from Ref. [38]. Copyright by the American Institute of Physics.

the excitation light. One may note that, contrary to spin orientation processes, this effect is not resonant. A large degree of polarization of light emitted by porous silicon has been indeed observed for energy differences between detection and excitation as large as $1 \mathrm{eV}$.

\subsubsection{Impurity in a spherical microcrystal}

We consider an impurity in a semiconductor microcrystal embedded in a low dielectric constant medium. The impurity dipole field polarizes the semiconductor and the dielectric medium. Both media create on the dipole a reactive field. As a result, the inductive field is the internal field of the microcrystal plus the reactive field. The local field becomes

$$
E_{\mathrm{loc}}=\frac{3}{\frac{n_{\text {int }}^{2}}{n_{\text {ext }}^{2}}+2+\frac{2 \alpha}{R^{3}}\left(\frac{n_{\text {int }}^{2}}{n_{\text {ext }}^{2}}-1\right)} E_{\mathrm{exc}},
$$

where $\alpha$ is the polarizability of the dipole and $R-$ the radius of the sphere. The dipole is assumed to be in the center of the sphere.

The internal field acting on the impurity dipole is reduced compared to the external field. In contradiction with the conclusions of Bhargava et al. [39] who studied the rate of spontaneous emission of $\mathrm{Mn}^{2+}$ in $\mathrm{ZnS}$, one expects the radiative lifetime of the impurity to be increased in microcrystals with respect to the bulk. 


\subsubsection{Quantum well}

In a QW or a very thin film, contributions to spontaneous emission from guided modes can be ignored and the spontaneous emission rate is only controlled by the free modes $[4,40,41]$. The refractive index of the external medium modifies differently dipoles parallel and perpendicular to the interfaces. For a parallel dipole there is no depolarizing field because the parallel component of the electric field is continuous across the boundaries of the film. The emission rate is mainly affected by the dependence on the refractive index of the electric field operator and the density of final states

$$
\frac{1}{\tau_{\mathrm{R}}}=\frac{n_{\mathrm{ext}}}{n_{\mathrm{int}}} \frac{1}{\tau_{\mathrm{R}}^{0}} \text {. }
$$

The emission rate of a perpendicular dipole is more affected because the vertical components of the dielectric field are discontinuous at the boundaries. When both interfaces are taken into account, we find that the electric field amplitude in the quantum well is reduced by a factor $\left(n_{\text {ext }} / n_{\text {int }}\right)^{2}$. The radiative lifetime becomes

$$
\frac{1}{\tau_{\mathrm{R}}}=\left(\frac{n_{\mathrm{ext}}}{n_{\mathrm{int}}}\right)^{5} \frac{1}{\tau_{\mathrm{R}}^{0}} .
$$

These predictions could be checked by measuring the selection rules with polarized light of the light exciton transition in a quantum well embedded in a low index medium.

TABLE I

Spontaneous rate of emission in a dielectric medium.

\begin{tabular}{c|c|c}
\hline Dipole & Nanocrystal & Quantum well \\
\hline $\begin{array}{c}\text { Electric } \\
n\left(\frac{n^{2}+3}{3}\right)^{2}\end{array}$ & Spherical & Dipole parallel \\
\hline Magnetic & $\left.\frac{3}{n_{\text {int }}^{2} / n_{\text {ext }}^{2}+2}\right)^{2} \frac{n_{\text {ext }}}{n_{\text {int }}}$ & $\frac{n_{\text {ext }}}{n_{\text {int }}}$ \\
$n^{3}$ & Spheroid & Dipole perpendicular \\
& $\frac{1}{3} \sum_{i}\left[\frac{1}{1+\left(n_{\text {int }}^{2} / n_{\text {ext }}^{2}-1\right) A_{i}}\right]^{2} \frac{n_{\text {ext }}}{n_{\text {int }}}$ & $\left(\frac{n_{\text {ext }}}{n_{\text {int }}}\right)^{5}$
\end{tabular}

\subsection{Coulomb interaction}

\subsubsection{Thin film or quantum well}

Coulomb interaction between electron and hole is very much screened in semiconductors because of the large value of the dielectric constant. As a consequence, excitons have small binding energies and large effective Bohr radius

$$
E_{\mathrm{ex}}=\frac{m e^{4}}{2 \varepsilon^{2} \hbar^{2}}, \quad a_{0}=\frac{\varepsilon \hbar^{2}}{m e^{2}},
$$

$m$ is the excitonic effective mass and $\varepsilon$ - the dielectric constant. 
The enhancement of the Coulomb interaction in a thin semiconductor film sandwiched by insulators was first pointed out by Keldysh $[25,26]$. This effect is caused by the reduction of the effective dielectric constant when the electric field penetrates the low dielectric constant barriers. The dielectric constants to use in Eq. (2.10) may be different from $\varepsilon=n^{2}$. For a film thickness $d \gg 40 \AA$ the Coulomb interaction in GaAs is determined by the static dielectric constant while for $d \ll 15 \AA$ the Coulomb interaction is determined by the optical dielectric constant [27].

The exciton binding energy $E_{\text {ex }}$ and the effective Bohr radius are calculated for a film of dielectric constant $\varepsilon$ sandwiched between two media of dielectric constants $\varepsilon_{1}$ and $\varepsilon_{2}$. We assume that $\delta=\frac{\varepsilon_{1}+\varepsilon_{2}}{2 \varepsilon} \ll 1$. In the limiting case of a film thickness $d$ such that $a_{0} \delta^{2} \ll d \ll a_{0}$, the binding energy is analytically given by

$$
E_{\mathrm{ex}}=-\frac{e^{2}}{\varepsilon d}\left(\ln \frac{d}{a_{0} \delta^{2}}-0.8\right)
$$

The exciton characteristic distance becomes

$$
a=\frac{1}{2} \sqrt{a_{0} d}
$$

A general calculation has been done more recently by Kumagai and Takagahara [27]. They show that in a $1 \mathrm{~nm}$ thick GaAs quantum well embedded in a medium of dielectric constant $\varepsilon=5$, the binding energy is multiplied by 30 and the Bohr radius divided by 40 with respect to the bulk values.

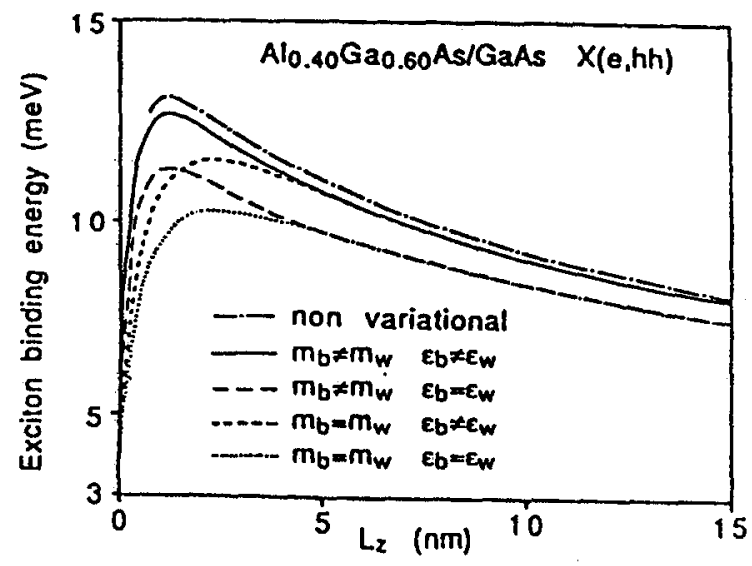

Fig. 6. Heavy-hole exciton binding energy as a function of quantum well width in $\mathrm{Al}_{0.40} \mathrm{Ga}_{0.60} \mathrm{As} / \mathrm{GaAs}$ quantum wells for different approximations to the barrier parameters. Reproduced from Ref. [28] with the author's permission.

Tran Thoai et al. [28] have compared the contribution of different masses and different dielectric constants in the quantum well and in the barrier. Figure 6 shows the result of their calculations. A change in the binding energy due to the discontinuity of the masses at the interfaces occurs only at well widths where the exciton leaks out into the barriers. On the contrary, the electrostatic interaction manifests itself over larger distances than the exciton Bohr radius. 
The increase in the exciton binding energy is important for its stability, especially at room temperature. On the other hand, the change of Bohr radius has a great effect on the optical properties since in thin films, the oscillator strength is inversely proportional to the square of the effective Bohr radius. Only few experiments have checked these predictions. Bagaev et al. [42] have studied thin CdTe films deposited on a $\mathrm{MgF}_{2}$ substrate. They measured the exciton peak energy as a function of film thickness. From the expected band gap variation they deduced an exciton binding energy which can exceed the bulk one by an order of magnitude. More recently, by varying the dielectric environment in $\mathrm{PbI}_{4}$-based layer-type perovskite compounds, Hong et al. [43] have directly demonstrated the contribution of the dielectric confinement to the exciton binding energy in "natural quantum well" semiconductors. Kumagai and Takagahara [27] proposed to consider a GaAs quantum well sandwiched by $\mathrm{ZnSe}$ barrier layers. ZnSe seems indeed to be one of the most favorable materials since its lattice constant nearly matches the GaAs one and its dielectric constant is smaller. To our knowledge no experiment has been done in this material up to now.

\subsubsection{Nanocrystals}

Takagahara [29] has considered the effects of dielectric confinement on excitonic states in semiconductor quantum dots in the strong confinement regime. The effects appear more pronounced than in quantum well and quantum wire structures because of more probable penetration of the electric force lines into the surrounding medium having a smaller dielectric constant. The increasing trend is also more pronounced for the smaller crystallites. The dielectric confinement has a significant effect on the exciton energy and cannot be treated as a small perturbation (see Fig. 7). For CdSe NCs of $25 \AA$ radius embedded in silicate glass one calculates that the first optical transition is shifted by $50 \mathrm{meV}$ to low energy

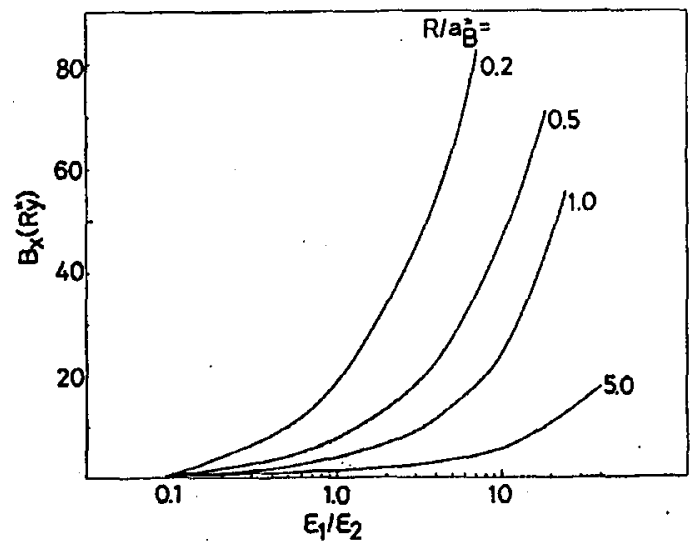

Fig. 7. Binding energy of the lowest exciton state as a function of the dielectric-constant ratio $\varepsilon_{1} / \varepsilon_{2}$ for a fixed value of the electron-hole mass ratio, $m_{\mathrm{e}} / m_{\mathrm{h}}=0.2$ and different values of the quantum dot radius. Reproduced from Ref. [29] with the author's permission. 
because of the dielectric confinement. The enhancement factor of the oscillator strength is larger than that for the case of a quantum well having a thickness of the same magnitude as the quantum dot diameter. In the weak confinement regime, for CdSe NCs of $100 \AA$ radius, the oscillator strength is multiplied by 1.5 by the dielectric confinement (see Fig. 8).

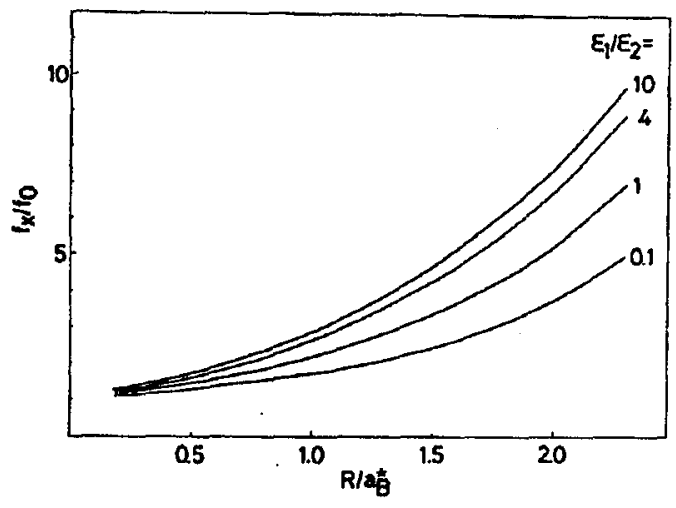

Fig. 8. Normalized oscillator strength $f_{x} / f_{0}$ for the lowest exciton state as a function of the quantum dot radius for a fixed value of the electron-hole mass ratio, $m_{c} / m_{\mathrm{h}}=0.2$ and different values of the dielectric constant ratio $\varepsilon_{1} / \varepsilon_{2}$. Reproduced from Ref. [29] with the author's permission.

\section{A small object close to a dielectric interface ${ }^{\dagger}$}

\subsection{Coupling with light}

\subsubsection{Intensity}

The Fermi golden rule which gives the rate of emission of an electric dipole at frequency $\omega_{\mathrm{a}}$ for a $(j=x, y, z)$ polarization

$$
\Gamma_{j}=\frac{2 \pi}{\hbar^{2}} \sum_{f}\left|\left\langle 0\left|\mu E_{j}\right| f\right\rangle\right|^{2} \delta\left(\omega-\omega_{\mathrm{a}}\right)
$$

can be formally summed up on the complete set of one-photon states $|f\rangle$ :

$$
\Gamma_{j}=\frac{2 \pi}{\hbar^{2}} \mu^{2}\left\langle 0\left|E_{j}\right| 0\right\rangle \rho\left(\omega_{\mathrm{a}}\right) \text {. }
$$

The summation leads to an expectation value which is identical to the vacuum electric-field fluctuation except for the insertion of the frequency-delta function in the integrand. The vacuum field fluctuation is changed if it is evaluated not in free space but in an environment where there are material bodies, for example metallic or dielectric media. In such systems the spontaneous emission rate can be either increased or decreased relative to its free space value.

The main results are summarized in Table II. 

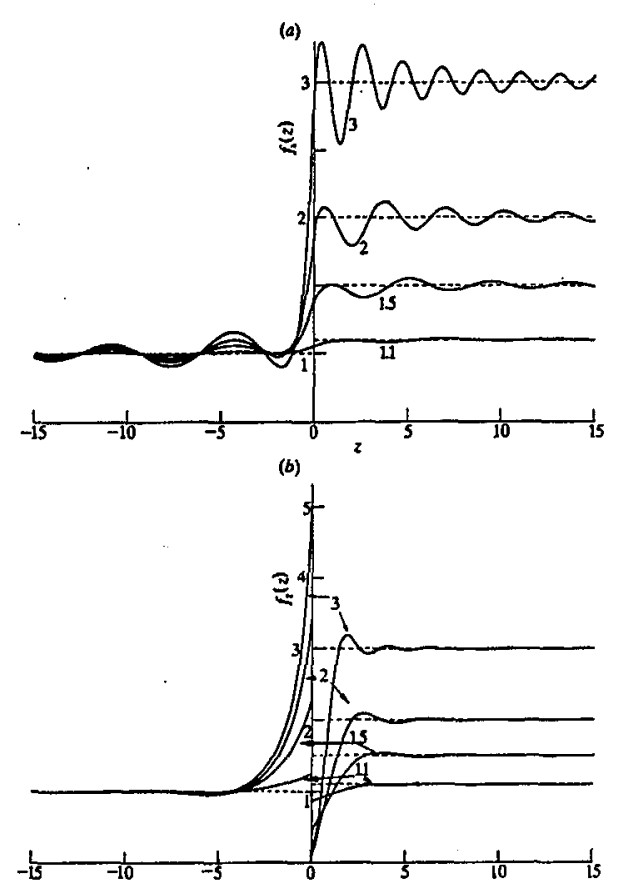

Fig. 9. Spatial dependence of the vacuum electric fluctuations in the vicinity of the dielectric/free space interface: (a) polarization parallel to the interface, (b) polarization perpendicular to the interface. The abcissa is $Z=2 \omega z / c$ where $\omega$ and $z$ are the frequency and the distance to the interface of the dipole, respectively. $c$ is the speed of light in vacuum. Reproduced with permission from Ref. [8]. Copyright by the Royal Society.

Khosravi and Loudon [8] have calculated the spatial dependence of the vacuum electric field fluctuation in the vicinity of a dielectric/free space interface (see Fig. 9). Inside the dielectric, oscillations in the spontaneous emission rate are due to the interference of direct and reflected waves. Close to the interface, the behavior of dipoles parallel and perpendicular to the interface are quite different. The rate of emission of a dipole parallel to the interface is always larger than the free space value. On the other hand, there is a region close to the interface where the emission rate of a dipole perpendicular to the surface is smaller than in free space. These results complete and confirm the results obtained in the case of a thin film embedded in a low index medium (see Sec. 2.1.5).

Outside the dielectric, oscillations due to the interferences of direct and reflected waves occur. Close to the interface and for both dipole orientations, a large increase in the spontaneous emission rate is observed. It corresponds to the possibility for the dipole to emit light in evanescent waves. Far from the interface, the emission rate is proportional to the refractive index of the medium. Recent experiments [44] have been done with Er-implanted silicate glass covered with a range of transparent liquids. The spontaneous emission rate of erbium ions around $1.54 \mu \mathrm{m}$ shows clear changes depending on the liquid refractive index and the er- 


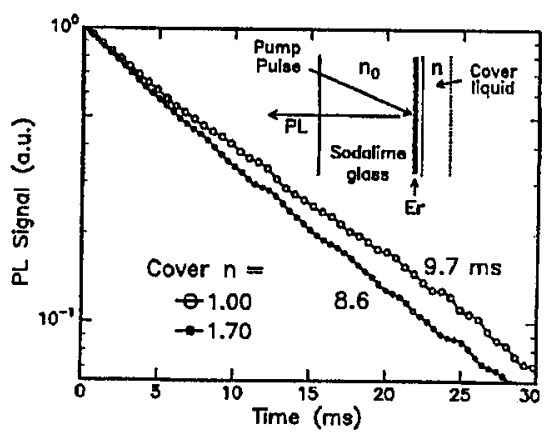

Fig. 10. Photoluminescence decay of the $1.537 \mu \mathrm{m}$ emission from erbium-implanted glass at a distance $20 \mathrm{~nm}$ of the surface. The solid data points were measured with a methylene-iodide film $(n=1.70)$ covering the surface, the open data points in air. Reproduced from Ref. [44] with the authors' permission.

bium distance to the interface. Figure 10 shows the photoluminescence decay for two situations, when the glass is in air or when it is covered with a high refractive index film $(n=1.70)$. In this last case, erbium ions are more coupled with light because they can emit waves which are evanescent in glass and propagate in the film. From the data taken with different cover liquids, it is possible to deduce the radiative transition rates of erbium.

\subsubsection{Directions of emission}

Lukosz and Kunz [7] have considered the situation in which a dipole is perpendicular to the interface.

3.1.2.1. Dipole located in the low refractive index medium. Figure 11 shows the angular distribution of emitted power for dipoles located at the interface in the low refractive index medium. Most of the power is radiated into the denser medium with a sharp peak at the critical angle which corresponds to total reflection i.e. to emission in evanescent waves into the rarer medium. Figure 12 shows the angular power distribution for dipoles located at different distances from the interface. The transmitted power in the denser medium is the same for angles smaller than the critical angle. It is reduced for angles larger than the critical angle when the distance of the dipole to the interface is increased.

3.1.2.2. Dipole located in the high refractive index medium. Figure 13 shows the radiation pattern of a dipole lying on the interface in the denser medium. The electric dipole emission has a peak at the critical angle.

From both Figs. 11 and 13 we conclude that a magnetic or an electric dipole lying very close to the interface radiates predominantly into the denser medium, regardless whether the dipole itself lies in the rarer or the denser medium.

The influence of a dielectric interface on the fluorescence decay of europium ions has been studied by Drexhage [15]. The absorption and emission of evanescent photon by dye molecules was investigated by Carniglia et al. [13] and Lieberherr et al. [16]. The experimental results are in very good agreement with the theory. 
Fig.11

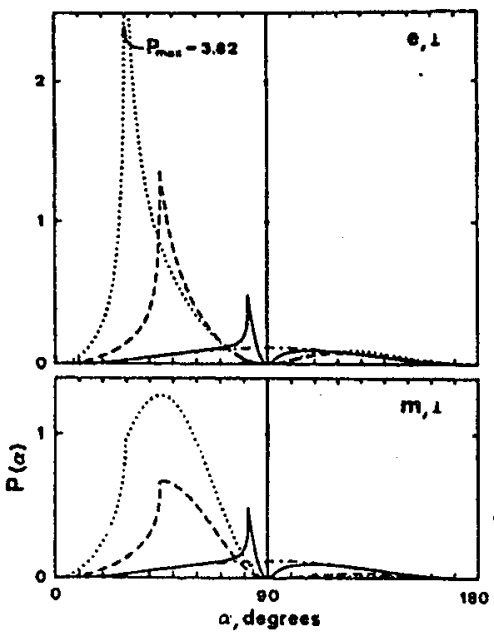

Fig. 12
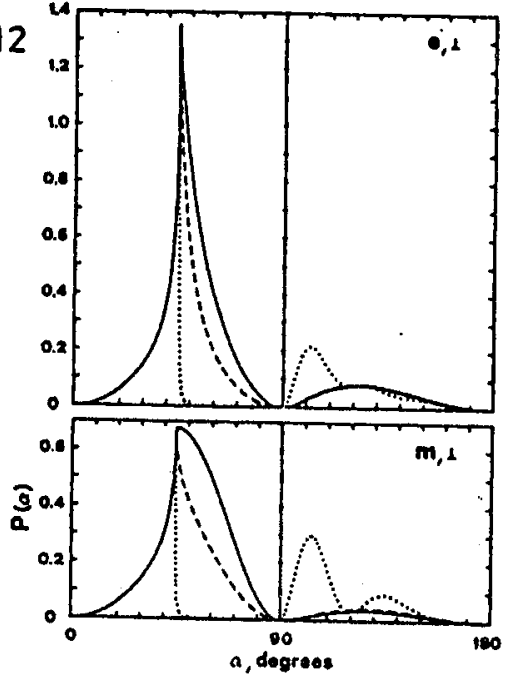

Fig. 13
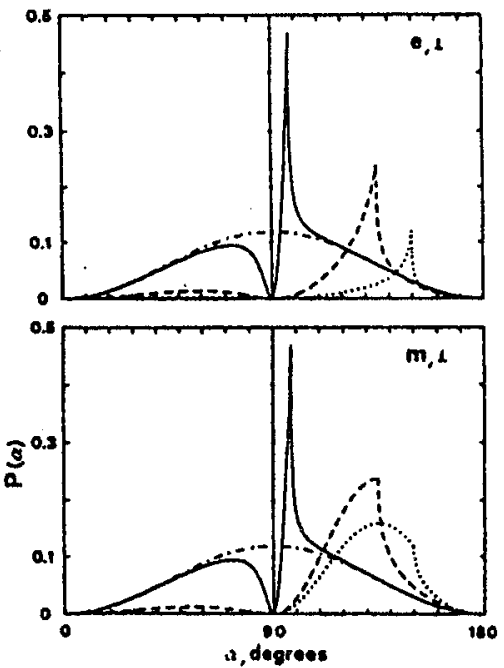

Fig. 11. Angular distribution of emitted power vs. angle for electric (e) and magnetic (m) dipoles located on the interface for relative refractive indices $n=1.01(-), n=\sqrt{2}$ $(---)$ and $n=2(\cdots)$ ). Curves (----) give the angular distribution in an unbounded medium $(n=1)$. Reproduced with permission from Ref. [7]. Copyright by the Optical Society of America.

Fig. 12. Angular distribution of emitted power for electric (e) and magnetic (m) dipoles at distances $z=0(-), z=\lambda_{1} / 4 \pi(-\cdots)$ and $z=\lambda_{1}(\cdots)$ from the interface. $\lambda_{1}$ is the wavelength of light in medium 1 . The relative refractive index is $n=\sqrt{2}$. Reproduced with permission from Ref. [7]. Copyright by the Optical Society of America.

Fig. 13. Radiation patterns as in Fig. 11 but for $n=1 / 1.01(-), n=1 / \sqrt{2}(-\cdots)$ and $n=1 / 2(\cdots \cdots)$. Reproduced with permission from Ref. [7]. Copyright by the Optical Society of America. 


\subsection{Coulomb interaction}

\subsubsection{Quantum well}

The exciton is made from an electron and a hole. They both have an image in the external medium. Let us call $\varepsilon_{\text {ext }}$ and $\varepsilon_{\text {int }}$ the dielectric constants in the external and internal media respectively. A charge $q$ in the internal medium has an image $q^{\prime}$ in the external medium given by

$$
q^{\prime}=\frac{\varepsilon_{\text {int }}-\varepsilon_{\mathrm{ext}}}{\varepsilon_{\text {int }}+\varepsilon_{\mathrm{ext}}} q .
$$

If the external medium has a low dielectric constant, the charge and the image charge have the same sign. The interaction between one particle and its image is repulsive and the particle self-energy increases. On the other hand, the electron is attracted by the hole and the hole image in the external medium so that the exciton binding energy is enhanced. These conclusions are in agreement with the previous conclusions which we obtained for a quantum well or a thin film in a medium of a low dielectric constant (Sec. 2.2.1).

A few experiments have been done on quantum wells close to the interface semiconductor/air [20-24]. In GaAs/GaAlAs quantum wells, Moison et al. [20] observed a dramatic decrease in luminescence intensity and a red shift of the luminescence line when the top barrier thickness was reduced (see Fig. 14). In GaAs/GaInAs quantum wells, however, Dreybrodt et al. [22] observed a blue shift of the line (see Fig. 15). Very likely, for short distances, tunneling to surface states occurs. This process may be partly responsible for the shift of the spectrum. In order to check the variation of self-energy and exciton binding energy, other experiments are needed in more controlled conditions.

TABLE II

Spontaneous rate of emission and Van der Waals energy of an exciton close to a dielectric interface.

\begin{tabular}{|c|c|c|c|}
\hline Far from the surface & $\begin{array}{l}\text { - Oscillations } \\
\text { distance } \\
\text { - The directior } \\
\text { on the dist }\end{array}$ & $\begin{array}{l}\text { a function of the } \\
\text { of emission depend } \\
\text { ice }\end{array}$ & \\
\hline \multirow[t]{2}{*}{$\begin{array}{l}\text { Close to the surface } \\
\text { in the denser medium }\end{array}$} & $\begin{array}{l}\text { Dipole parallel } \\
\text { weak change }\end{array}$ & $\begin{array}{l}\text { Dipole perpendicular } \\
\text { large decrease }\end{array}$ & \multirow{3}{*}{$\begin{array}{l}\text { The quantum } \\
\text { well radiates } \\
\text { predominantly } \\
\text { in the denser } \\
\text { medium with a } \\
\text { maximum of } \\
\text { emission at the } \\
\text { critical angle }\end{array}$} \\
\hline & \multicolumn{2}{|c|}{$\begin{array}{l}\text { - The self-energy and the binding } \\
\text { energy increase }\end{array}$} & \\
\hline $\begin{array}{l}\text { Close to the surface } \\
\text { in the rarer medium }\end{array}$ & \multicolumn{2}{|c|}{$\begin{array}{l}\text { - Increase due to the emission in } \\
\text { the evanescent waves } \\
\text { - The self-energy and the binding } \\
\text { energy decrease }\end{array}$} & \\
\hline
\end{tabular}



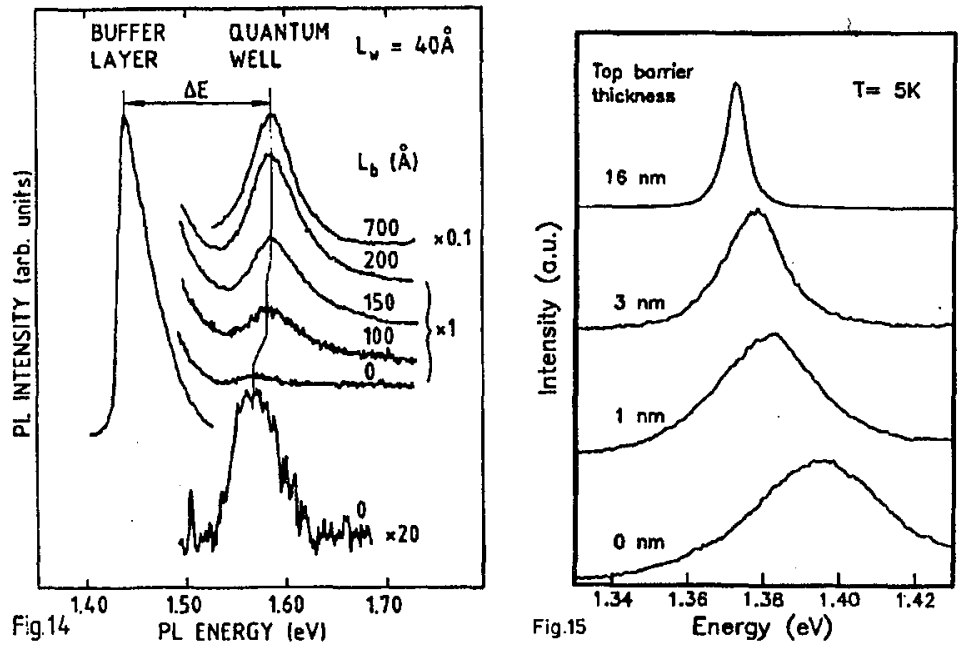

Fig. 14. Photoluminescence spectra of a near surface $\mathrm{GaAs} / \mathrm{Ga}_{0.7} \mathrm{Al}_{0.3}$ As quantum well with width $L_{\mathrm{w}}=40 \AA$ and different barrier thicknesses, $L_{\mathrm{b}}$. Reproduced from Ref. [20] with the authors' permission.

Fig. 15 Photoluminescence spectra of a near surface $5 \mathrm{~nm} \mathrm{Ga0.80} \mathrm{In}_{0.20}$ As/GaAs quantum well with different barrier thicknesses. Reproduced with permission from Ref. [22]. Copyright by the Editions de Physique.

\section{A small object close to a mirror ${ }^{\dagger}$}

\subsection{Coupling with light}

\subsubsection{Dipole or quantum well}

4.1.1.1. Thick mirror. Chance et al. [10] have studied the dipole emission near a metallic interface. Figure 16 shows the lifetime and the quantum yield of a dipole versus distance from a silver mirror. As for a dielectric interface, the rate of the spontaneous emission oscillates with distance because of the interferences between direct and reflected waves. The contrast is larger than in the case of a dielectric interface. Far from the mirror, the quantum yield is not equal to 1 because the mirror is not perfectly reflecting. Close to the mirror both radiative and nonradiative decay are modified. The spontaneous emission rate is evaluated by considering the dipole and its image. For a dipole parallel to the interface the image is reversed and the emission rate of the resulting quadrupole becomes zero at the interface. For a dipole normal to the interface, the image dipole is not reversed and the emission rate is multiplied by a factor 2 at the interface. On the other hand, the dipole-dipole interaction between the dipole and its image leads to a nonradiative transfer to the surface-plasmon mode. This interaction decreases with distance as $1 / d^{3}$ for a bulk mirror. Phenomenologically, the imaginary part of the mirror refractive index is responsible for this nonradiative decay of the oscillating dipole. The energy transfer rate has a resonance near the surface-plasmon frequency i.e.

The main results are summarized in Table III. 
when the difference between the metal dielectric constant and the dielectric constant of the medium in which is embedded the dipole is equal to -2.25 . Note that, at resonance, energy transfer competes successfully with emission within $1000 \AA$ of the surface.

TABLE III

Spontaneous rate of emission and Van der Waals energy of an exciton close to a metallic interface.

\begin{tabular}{l|l|l}
\hline \hline $\begin{array}{l}\text { Thick mirror } \\
\text { Far from the surface }\end{array}$ & $\begin{array}{l}\text { - Oscillations as a function of the distance } \\
\text { - The directions of emission depend on the distance }\end{array}$ \\
\hline Close to the surface & $\begin{array}{l}\text { Dipole parallel } \\
\text { large decrease }\end{array}$ & $\begin{array}{l}\text { Dipole perpendicular } \\
\text { increase by factor 2 }\end{array}$ \\
\cline { 2 - 2 } & $\begin{array}{l}\text { - Nonradiative transfer to the surface plasmon } \\
\text { - The self-energy and the binding energy decrease }\end{array}$ \\
\hline $\begin{array}{l}\text { Metallic sphere or } \\
\text { metal-island thin film }\end{array}$ & $\begin{array}{l}\text { - Radiative and nonradiative coupling to the } \\
\text { plasmon modes }\end{array}$
\end{tabular}
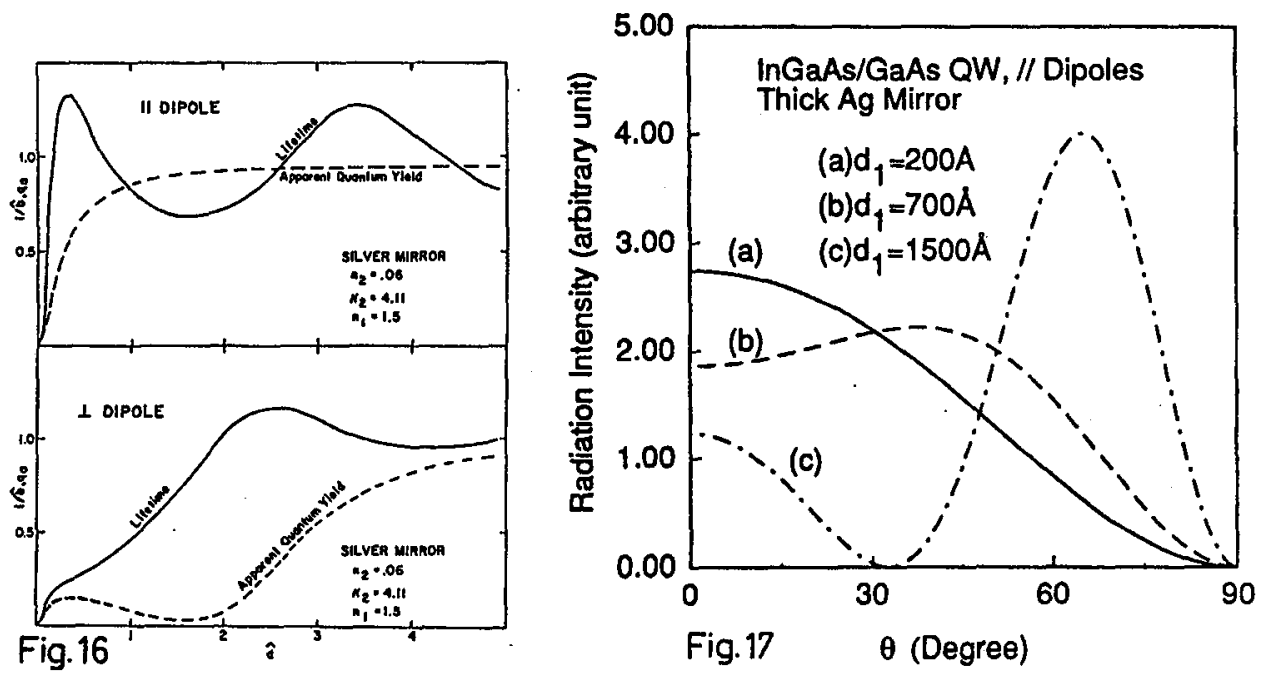

Fig. 16. Quantum yield $q_{\mathrm{a}}$ and lifetime $1 / \widehat{b}$ versus distance $\widehat{d}\left(d=2 \pi n_{1} d / \lambda\right)$ from a silver mirror $\left(n_{2}=0.06+\mathrm{i} 4.11\right)$. Reproduced from Ref. [10] with the authors' permission. Fig. 17. Radiation pattern of the parallel dipole component in a GaAs/GaAs quantum well at a distance $d_{1}$ from a thick silver mirror. Reproduced with permission from Ref. [21] (C 1993 IEEE).

The theory has been carefully compared with the results of experiments done on anthracene close to gold or aluminium mirrors [12] and europium complexes 
close to silver or gold mirrors $[14,15]$. Numerical calculations have been done by Huang et al. [21] for InGaAs/GaAs QWs close to a silver mirror. At the emission wavelength $(1 \mu \mathrm{m})$, the nonradiative transfer of a QW dipole located at $600 \AA$ of a silver mirror is still $20 \%$ of the radiative rate. The radiation pattern of the dipole is modified by the presence of the mirror. Figure 17 shows the calculated radiation pattern for a InGaAs/GaAs QW located at different distances from the mirror. For a QW located at $1500 \AA$ from the mirror, constructive interferences between direct and reflected waves contribute to a maximum of emission in a direction very different from the normal to the mirror. The conservation law holds for the QW exciton and the emitted photon momentum parallel to the QW interface. Depending on the emission direction, either with constructive or destructive interferences, the emission rate of an exciton with definite plane momentum is increased or decreased. Ilowever, the fast relaxation of excitons between different momentum states should prevent observing a variation of the radiative lifetime of excitons with the direction of emission.

4.1.1.2. Metal-island film. In a small metallic particle, a maximum of the internal field occurs at a frequency $\omega$ where the real part of the denominator in Eq. (2.6) vanishes, i.e. for

$$
\varepsilon(\omega)=1-\frac{1}{A}
$$

This condition corresponds to the excitation of the lowest order particle plasmon. It is represented by a dipolar distribution of surface charges oscillating at frequency $\omega$ [45]. Outside the particle, the total field distribution equals the applied field plus the field of the equivalent dipole located at its center. Then, there is a large enhancement of the field close to the metal particle. A similar situation is found in thin metal-island films. Glass et al. [46] have studied the optical properties of silver films overcoated with thin layers of dyes. Figure 18 shows the

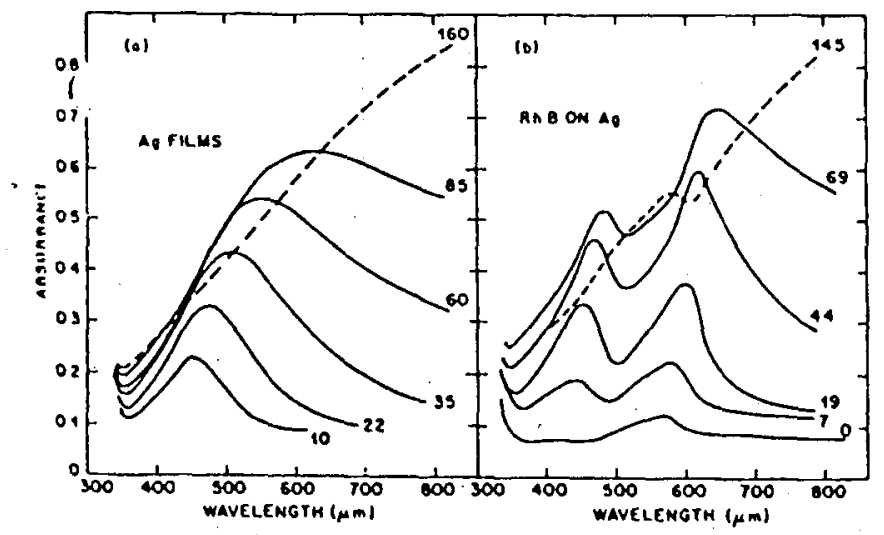

Fig. 18. Absorption spectra of wedge-shaped silver film at various thicknesses of the wedge shown in angstroms: (a) silver film alone, (b) silver film with overlayer of rhodamine B. Reproduced with permission from Ref. [46]. Copyright by the Optical Society of America. 


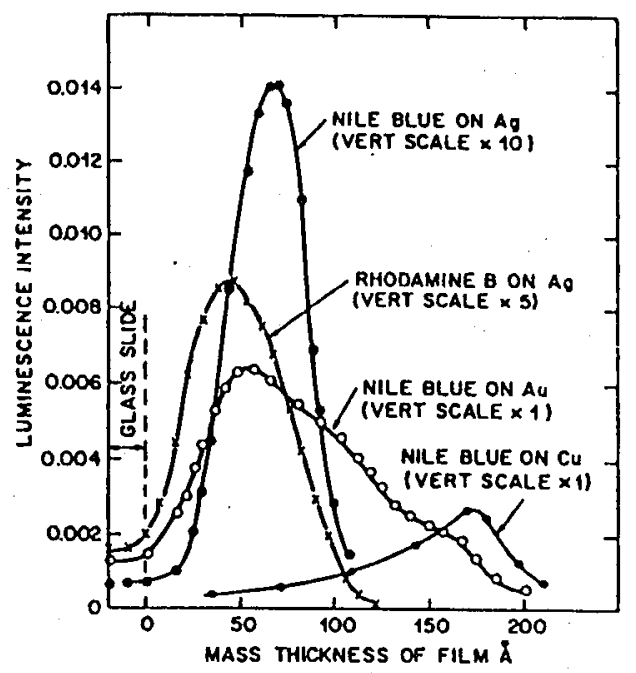

Fig. 19. Luminescence intensity of various dyes on wedge-shaped metal films for various thicknesses of the film. Reproduced with permission from Ref. [46]. Copyright by the Optical Society of America.

absorption of the films with and without an adsorbed dye monolayer. The coated film exhibits a characteristic doublet of absorption bands. The damping of the particle corresponds to enhanced absorption in the dye molecules. This enhanced absorption does not necessarily correspond to enhanced luminescence since radiative decay competes with nonradiative transfer to other excitation modes of the particle plasmon. Figure 19 shows that it is, nevertheless, possible to obtain a large enhancement of the luminescence intensity at a film thickness where the plasmon resonance frequency of the metal film coincides with the dye absorption. Nitzan and Brus [47] have suggested that the enhancement of luminescence intensity may be even larger if the molecule is not in contact with the metal. This results from the sharp dependence $1 / d^{3}$ of the nonradiative transfer in contrast to the mild dependence $1 /(d+a)^{3}$ of the field enhancement on the molecule located at a distance from $d+a$ of the center of the metallic sphere. To our knowledge, no systematic study of luminescence intensity has been done with NCs on metal-island films. The enhancement of luminescence light on thin metal films might help to study the optical properties of one isolated nanocrystal.

\subsection{Coulomb interaction}

\subsubsection{Quantum well}

In the vicinity of a metallic mirror, the charge and the image charge have opposite signs. Unlike the situation described in Sec. 3.2.1, the self-energy of the particles and the exciton binding energy decrease.

The self-energy shift of a decaying dipole in the vicinity of a mirror has been determined from the static dipole-dipole interaction [48]. In a quantum well one 
should expect similarly a shift

$$
\Delta E=-E_{\text {exc }}\left(\frac{a_{0}}{d}\right)^{3},
$$

where $E_{\text {exc }}$ and $a_{0}$ are the exciton binding energy and Bohr radius respectively. $d$ is the distance of the QW to the surface. This formula is valid only for distances $d$ larger than $a_{0}$ so that one can neglect the variation of the exciton binding energy.

No experiences have been done up to now to check these predictions.

\section{Conclusion}

We have shown that the light-exciton coupling as well as the exciton binding energy in a $\mathrm{QW}$ or a NC are modified by the environment. These modifications are of practical importance for the study of the optical properties of QWs and NCs and for their use in photoelectronic devices.

In most studies the QW is embedded in other semiconductor which has a high refractive index. The QW is not far from the semiconductor/air interface. For a distance $d$ to the interface smaller than $n \lambda / 4 \pi$, i.e. $1000-2000 \AA$, the rate of spontaneous emission with polarization perpendicular to the interface is very much reduced. This reduction affects the light-exciton transition which can be the lowest transition in InGaAs/GaAs quantum wells.

For both polarizations, the $\mathrm{QW}$ radiates predominantly towards the denser medium. In cases where the structure was grown on a substrate transparent to the light emitted by the QW, one could collect light with a higher efficiency from the substrate side.

It can be interesting to increase the binding energy of the exciton in the QW in order to increase its stability and oscillator strength. This happens for a range of distances to-the surface of the order of twice the Bohr radius of the exciton. However, it is not clear how, for such distances, the optical properties of the exciton are affected by a nonradiative transfer to the surface states.

In order to collect light efficiently one should avoid to locate the $\mathrm{QW}$ at distances lower than ' $1000-2000 \AA$ from a reflecting interface.

Similar conclusions are reached for small crystals. The rate of spontaneous emission of a microcrystal is enhanced when it is immersed in a medium of high refractive index medium. In order to study the optical properties of NCs, one should avoid locating them on a reflecting interface. However, on a metal-island thin film (mass thickness smaller than $100 \AA$ ), radiative coupling with the metallic plasma modes may compete efficiently with nonradiative coupling when the emission of light is resonant with the plasmon frequency: Such a situation can be found also in metal coated nanocrystals.

\section{Acknowledgment}

I am grateful to M. Combescot for her help and cooperation throughout this work. I would like to thank her specially for helpful comments about the binding energy of excitons close to a dielectric or metallic interface. 


\section{References}

[1] M. Purcell, Phys. Rev. 69, 681 (1946).

[2] G. Nienhuis, C.Th.J. Alkemade, Physica C 81, 181 (1976).

[3] W. Lukosz, Phys. Rev. B 22, 3030 (1980).

[4] E. Yablonovitch, T.J. Gmitter, R. Bhat, Phys. Rev. Lett. 61, 2564 (1988).

[5] R.J. Glauber, M. Lewenstein, Phys. Rev. A 43, 467 (1991).

[6] C.K. Carniglia, L. Mandel, Phys. Rev. D 3, 280 (1971).

[7] W. Lukosz, R.E. Kunz, J. Opt. Soc. Am. 67, 1607 (1977).

[8] H. Khosravi, R. Loudon, Proc. R. Soc. Lond. A 433, 337 (1991).

[9] H. Kuhn, J. Chem Phys. 53, 101 (1970).

[10] R.R. Chance, A. Prock, R. Silbey, in: Molecular Fluorescence and Energy Transfer near Interfaces, Vol. XXXVII of Advances in Chemical Physics, Eds. I. Prigogine, S. Rice, Wiley, New York 1978, p. 1.

[11] S. Haroche, D. Kleppner, Physics Today 42, 24 (1989) and references therein.

[12] G. Vaubel, H. Bässler, D. Möbius, Chem. Phys. Lett. 10, 334 (1971).

[13] C.K. Carniglia, L. Mandel, K.H. Drexhage, J. Opt. Soc. Am. 62, 479 (1972).

[14] K.H. Tews, Ann. Phys. 29, 97 (1973).

[15] K.H. Drexhage, in: Progress in Optics XII, Ed. E. Wolf, North-Holland, Amsterdam 1974, p. 165.

[16] M. Lieberherr, Ch. Fattinger, W. Lukosz, Surf. Sci. 189, 959 (1987).

[17] G.L.J.A. Rikken, Y.A.R.R. Kessener, Phys. Rev. Lett. 74, 880 (1995).

[18] Y. Yamamoto, R. Slusher, Physics Today 46, 66 (1993).

[19] For a review, see Confined Electrons and Photons. New Physics and Applications, NATO ASI Series B, Physics, Vol. 340, Eds. E. Burstein, C. Weisbuch, Plenum Press, New York 1995.

[20] J.M. Moison, K. Elcess, F. Houzay, J.Y. Marzin, J.M. Gérard, F. Barthe, M. Bensoussan, Phys. Rev. B 41, 12945 (1990).

[21] Z. Huang, C.C. Lin, D.G. Deppe, IEEE J. Quantum Electron. 29, 2940 (1993).

[22] J. Dreybrodt, A. Forchel, J.P. Reithmaier, J. Phys. IV, coll. C5, suppl. au J. Phys. II, 265 (1993).

[23] Y.-L. Chang, I.-H. Tan, Y.-H. Zhang, D. Bimberg, J. Merz, E. Hu, J. Appl. Phys. 74, 5144 (1993).

[24] J. Dreybrodt, F. Daiminger, J.P. Reithmaier, A. Forchel, Phys. Rev. B 51, 4657 (1995).

[25] L.V. Keldysh, JETP Lett. 29, 658 (1979).

[26] L.V. Keldysh, JETP Lett. 30, 224 (1979).

[27] M. Kumagai, T. Takagahara, Phys. Rev. B 40, 12359 (1989).

[28] D.B. Tran Thoai, R.Z. Zimmermann, M. Grundmann, D. Bimberg, Phys. Rev. B 42, 5906 (1990).

[29] T. Takagahara, Phys. Rev. B 47, 4569 (1993).

[30] W.A. Saunders, K.J. Vahala, H.A. Atwater, R.C. Flagan, J. Appl. Phys. 72, 806 (1992). 
[31] S. Juen, K. Überbacher, J. Baldauf, K.F. Lamprecht, R.A. Höpfel, Appl. Phys. Lett. 62, 1958 (1993).

[32] H.S. Zhou, H. Sasahara, I. IIonma, II. Komiyama, J.W. Iraus, Chem. Mat. 6, 1534 (1994) and references therein.

[33] P. Lavallard, M. Rosenbauer, T. Gacouin, Phys. Rev. A, to be published.

[34] J. Haus, H.S. Zhou, S. Takami, M. Hirasawa, I. IIonma, H. Komiyama, J. Appl. Phys. 73, 1043 (1993).

[35] H.S. Zhou, I. Honma, H. Komiyama, J.W. Haus, Phys. Rev. B 50, 12052 (1994).

[36] See for example L.D. Landau, E.M. Lifshitz, Electrodynamics of Continuous Media, Pergamon Press, Oxford 1962.

[37] P. Lavallard, R.A. Suris, Solid State Commun. 95, 267 (1995).

[38] D. Kovalev, M. Ben Chorin, J. Diener, F. Koch, Al.L. Efros, M. Rosen, N.A. Gippius, S.G. Tikhodeev, Appl. Phys. Lett. 67, 1585 (1995).

[39] R.N. Bhargava, D. Gallagher, X. Hong, A. Nurmikko, Phys. Rev. Lelt. 72, 416 (1994).

[40] H. Khosravi, R. Loudon, Proc. R. Soc. Lond. A 436, 373 (1992).

[41] S.T. Ho, S.L. McCall, R.E. Slusher, Opt. Lett. 18, 909 (1993).

[42] V.S. Bagaev, N.A. Bagaev, A.G. Poiarkov, Salashchenko, V.B. Stopachinskii, in: Proc. 17th Int. Conf. on the Physics of Semiconductors, 1984, Ed. J.D. Chadi, Springer Verlag, New York 1984, p. 371.

[43] X. Hong, T. Ishihara, A.V. Nurmikko, Phys. Rev. B 45, 6961 (1992).

[44] E. Snoeks, A. Lagendijke, A. Polman, Phys. Rev. Lett. 74, 2459 (1995).

[45] A. Wokaun, in: Solid State Phys. 38, 224 (1984).

[46] A.M. Glass, P.F. Lia, J.G. Bergman, D.H. Olson, Opt. Lett. 5, 368 (1980).

[47] A. Nitzan, L.E. Brus, J. Chem. Phys. 74, 5321 (1981).

[48] For a review on radiating atoms in confined space, see D. Meschede, Phys. Rep. 211, 201 (1992). 